\title{
BUNDLE-LIKE FOLIATIONS WITH KÄHLERIAN LEAVES ${ }^{1}$ \\ BY
}

RICHARD H. ESCOBALES, JR.

Dedicated to my father

\begin{abstract}
For bundle-like foliations with Kählerian leaves a certain function $f$ is studied and its Laplacian along a leaf is computed. From this computation one obtains geometric conditions which guarantee the integrability of the distribution orthogonal to that determined by the leaves. When the leaves are compact, the key condition needed to guarantee the integrability of this orthogonal distribution can be interpreted as a condition on the first Chern class of each of the leaves.
\end{abstract}

Introduction. Given a bundle-like foliation with Kählerian leaves under what conditions is the distribution orthogonal to that determined by the leaves integrable? In this paper we give two theorems which provide sufficient conditions for the integrability of this orthogonal distribution. The key condition in one of the theorems is a condition on the Ricci curvature of the leaves of the bundle-like foliation. If the leaves of the bundle-like foliation are compact, the condition can be interpreted as a condition on the first Chern class of the leaves of this foliation.

In $\S 1$, we introduce terminology, definitions and preliminary results needed in later sections. In $\$ 2$, we study a function, $f$, whose vanishing implies that the distribution orthogonal to the leaves of the distribution determined by the bundle-like foliation is integrable. In particular, we develop a formula for the Laplacian of this function along a leaf of the given bundle-like foliation. In $\$ 3$ we give the statements and proofs of the main results of the paper. We conclude the paper with an example which illustrates the necessity of the assumption on the Ricci curvature or first Chern class of the leaves. Unless otherwise indicated, we assume a differentiability class of at least $C^{\infty}$.

The results obtained in this paper are analogues of an earlier result which appeared in this journal [2]. The work was done while the author was on sabbatical leave from Canisius College. He is grateful to Professors Graver and Church of Syracuse University for their hospitality, to Dr. Glen Castore and Professor AbiKhuzam who initiated a seminar on holomorphic curves and to Professor H. Blaine Lawson for a conversation about an earlier version of this work.

1. Let $M$ be a complete connected manifold with foliation $\mathfrak{T}$ of codimension $q$. Then $\mathcal{V}$ may be defined by a maximal family of $C^{\infty}$ submersions $f_{\alpha}: U_{\alpha} \rightarrow f_{\alpha}\left(U_{\alpha}\right) \subset R^{q}$,

Received by the editors June 18, 1981 and, in revised form, March 3, 1982.

1980 Mathematics Subject Classification. Primary 53C15, 53C55; Secondary 57R30.

${ }^{1}$ The results of this paper were presented at the special session on differential geometry and ergodic theory at the AMS meeting held at the.University of Massachusetts in October 1981. 
where $\left\{U_{\alpha}\right\}_{\alpha \in \Lambda}$ is a maximal open cover of $M$ and where for each $p \in U_{\alpha} \cap U_{\beta}$, there is a local $C^{\infty}$ diffeomorphism, $\phi_{\beta \alpha}^{p}$, of $R^{q}$ so $f_{\beta}=\phi_{\beta \alpha}^{p} \circ f_{\alpha}$ in some neighborhood $U_{p}$ of $p$. The foliation $\mathcal{V}$ is bundle-like if each of the submersions $f_{\alpha}$ are Riemannian submersions from $U_{\alpha}$ onto their images $f_{\alpha}\left(U_{\alpha}\right)$. In this case, the local diffeomorphisms $\phi_{\beta \alpha}^{p}$ are local isometries from $f_{\alpha}\left(U_{p}\right)$ to $f_{\beta}\left(U_{p}\right)$. In general, the metric on $f_{\alpha}\left(U_{\alpha}\right)$ is not the flat metric. While we shall want our foliation to be bundle-like later in the paper, we do not need this condition in the present section.

From [5, pp. 2-3], we know for $p$ and $p^{\prime}$ in $U_{\alpha} \cap U_{\beta}, \phi_{\beta \alpha}^{p}=\phi_{\beta \alpha}^{p^{\prime}}$ on $f_{\alpha}\left(U_{p} \cap U_{p^{\prime}}\right)$. Observe that a tangent vector $\mathcal{V}$ belongs to the tangent space of the distribution at $p$, $\mathfrak{V}_{p}$, if and only if $f_{\alpha^{*} p} V=0$ or $V \in \operatorname{ker} f_{\alpha^{*} p}$. If $L$ is a leaf of $\mathscr{V}$ and $U$ is any open set of $M$, then each connected component of $U \cap L$ is called a plaque.

Now fix a Riemannian metric on $M$. Then the metric determines an orthogonal bundle, $\mathcal{H}$, to $\mathscr{T}$ in $T M$ where $T M$ is the tangent bundle of $M$. Thus, $T M=\mathcal{H}+\mathscr{V}$. A tangent vector $E$ on $M$ is called horizontal if $\mathcal{H} E=E$; the tangent vector $E$ is called vertical if $\mathfrak{V} E=E$. Here $\mathcal{H} E$ and $\mathfrak{V} E$ denote the obvious projections. A horizontal vector field $X$ on $U$ is called $f_{\alpha}$ basic if $f_{\alpha^{*}} X$ is a well-defined vector field on $f_{\alpha}\left(U_{\alpha}\right)$. In our earlier paper on bundle-like foliations in this journal [2], the following result was established.

Proposition 1.1. On $U_{\alpha} \cap U_{\beta}$ a horizontal vector field $X$ is $f_{\alpha}$ basic if and only if $X$ is $f_{\beta}$ basic.

Because of this result, we can speak of an $f_{\alpha}$ basic vector field on some open set $U$ as simply a basic vector field on $U$ or as a local basic vector field. If $V$ is any vertical vector field and $X$ is a basic vector field on $U_{\alpha}$, then $f_{\alpha^{*}}[X, V]=\left[f_{\alpha^{*}} X, f_{\alpha^{*}} V\right]=0$. This proves the first half of the following

LEMMA 1.2. (a) If $X$ is a basic vector field and $V$ is a vertical vector field on some open set $U_{\alpha}$, then $[X, V]$ is a vertical vector field on $U_{\alpha}$.

(b) If $X$ and $Y$ are basic vector fields on some open set $U_{\alpha}$, then $\mathcal{H}[X, Y]$ is a basic vector field on $U_{\alpha}$.

Proof of $1.2(\mathrm{~b})$. To see this, observe $[X, Y]=\mathcal{H}[X, Y]+\mathscr{V}[X, Y]$. Now $f_{\alpha^{*}}[X, Y]=\left[f_{\alpha^{*}} X, f_{\alpha^{*}} Y\right]$. But $f_{\alpha^{*}}[X, Y]=f_{\alpha^{*}} \mathcal{H}[X, Y]$, since $f_{\alpha^{*}} \mathscr{V}[X, Y]=0$. This completes the proof.

REMARK 1.3. Both of these results are well known in the case when $f_{\alpha}$ is a Riemannian submersion [6], a hypothesis we do not require here.

Suppose that the leaves of $\mathfrak{V}$ are complex manifolds and suppose that there exists a tensor $J$ on $M$ of tensor type $(1,1)$ so that when restricted to any plaque, $J$ is the complex structure for that plaque.

Definition 1.4. A basic vector field $X$ defined on some open set $U$ of $M$ respects the complex structure of the leaves provided for each vertical vector field $V$, $[X, J V]=J[X, V]$.

Now let $X$ and $Y$ be local basic vector fields which respect the complex structure of the leaves. If $V$ is any vertical vector field, it follows from the Jacobi identity that

$$
[[X, Y], J V]=[X,[Y, J V]]-[Y,[X, J V]] \text {. }
$$


Since $X$ and $Y$ respect the complex structure of the leaves, we have

$$
[[X, Y], J V]=[X, J[Y, V]]-[Y, J[X, V]] \text {. }
$$

By Lemma 1.2(a) $[Y, V]$ and $[X, V]$ are vertical, so

$$
[[X, Y], J V]=J[X,[Y, V]]-J[Y,[X, V]]=J[[X, Y], V] \text {. }
$$

Now by Lemma 1.2(b), $\mathcal{H}[X, Y]$ is basic when $X$ and $Y$ are basic, so

$$
[\mathcal{H C}[X, Y], J V]=J[\mathcal{H}[X, Y], V] \text {. }
$$

Subtracting (4) from (3) we get

$$
[\mathscr{V}[X, Y], J V]=J[\mathcal{V}[X, Y], V]
$$

Formula (5) has the following geometric interpretation.

Proposition 1.5. Let $X$ and $Y$ be basic vector fields on some open set $U$ of $M$ which respect the complex structure of the leaves of $\mathcal{V}$. Then when $\mathcal{V}[X, Y]$ is restricted to a plaque of $\mathfrak{V}, \mathfrak{V}[X, Y]$ is an infinitesimal automorphism of the complex structure of that plaque.

Proof. This is immediate from formula (5).

2. For the remainder of the paper suppose that:

(A) The foliation $\mathcal{V}$ is bundle-like with respect to the metric on $M$.

(B) The metric and connection on $M$ when restricted to any leaf of $\mathcal{V}$ gives rise to a Kähler structure on the leaf.

(C) There exists a tensor $J$ of tensor type $(1,1)$ on $M$ so that when restricted to any plaque of $\mathscr{V}, J$ is the complex structure of that plaque.

(D) Local basic vector fields respect the complex structure of the leaves.

We adopt the following conventions for this and the next section:

$1 \leqslant i, j \leqslant q$ where $q$ is the codimension of $\mathcal{T}$;

$1 \leqslant \alpha, \beta \leqslant n-q$ where $n-q$ is the dimension of each leaf of $\mathfrak{V}$.

For Greek subscripts only we use the Einstein summation convention on repeated indices.

Let $\left\{X_{1}, X_{2}, \ldots, X_{q}\right\}$ be any orthonormal frame of $\mathcal{H}$ at $p \in M$. Let

$$
f(p)=\sum_{i, j}\left\langle\bigvee\left[X_{i}, X_{j}\right], ף\left[X_{i}, X_{j}\right]\right\rangle
$$

where $\langle$,$\rangle is the Riemannian metric on M$. This function is independent of the choice of frame of $\mathcal{H}$ and so is well defined on all of $M$. Since $\mathcal{V}$ is bundle-like, the orthonormal frame can be chosen to consist of basic vector fields $\left\{X_{1}, X_{2}, \ldots, X_{q}\right\}$ defined on some open set $U$ of $M$. Observe this is where the bundle-like hypothesis comes into play. If $L$ is any leaf of $\mathscr{V}$, then $\mathscr{V}\left[X_{i}, X_{j}\right]$, when restricted to any plaque of $U \cap L$, is an infinitesimal automorphism of the complex structure of that plaque by Proposition 1.5.

Denote the complexification of $\mathscr{V}$ by $\mathcal{V}^{C}$ and set

$$
W_{i j}^{+}=\frac{1}{2}\left(ף\left[X_{i}, X_{j}\right]-\sqrt{-1} ף\left[X_{i}, X_{j}\right]\right)
$$


Then $W_{i j}^{+}$is a vector field defined on $U$. On each plaque of $U \cap L, W_{i j}^{+}$is the holomorphic vector field corresponding to the infinitesimal automorphism of the complex structure $\mathfrak{V}\left[X_{i}, X_{j}\right]$ determined by the well-known isomorphism [4, Chapter IX, Proposition 2.11]. On each plaque $W_{i j}^{+}$is of type $(1,0)$ and setting $W_{i j}^{-}=\overline{W_{i j}^{+}}$, one has $W_{i j}^{-}$is of type $(0,1)$. If we select coordinate fields $\left\{\partial / \partial z^{\alpha}, \partial / \partial \bar{z}^{\bar{\alpha}}\right\}$ for $1 \leqslant \alpha$, $\bar{\alpha} \leqslant n-q$ for a plaque of $U \cap L$, then

$$
ף\left[X_{i}, X_{j}\right]=W_{i j}^{\alpha} \frac{\partial}{\partial z^{\alpha}}+W_{i j}^{\bar{\alpha}} \frac{\partial}{\partial \bar{z}^{\bar{\alpha}}}
$$

where $W_{i j}^{\alpha}$ are the components of $W_{i j}^{+}$relative to $\left\{\partial / \partial z^{\alpha}\right\}_{1 \leqslant \alpha \leqslant n-q}$. Extend $\langle$,$\rangle to$ $\mathcal{V}^{C}$ and denote this extension by $g$. If $g_{\alpha \bar{\beta}}=g\left(\partial / \partial z^{\alpha}, \partial / \partial \bar{z}^{\bar{\beta}}\right)$, then

$$
f(p)=\sum_{i, j} 2 g_{\alpha \beta}-W_{i j}^{\alpha} W_{i j}^{\bar{\beta}}
$$

Following essentially Yano and Bochner [9, formula 8.53 on p. 133], we obtain

$$
\left(\Delta_{\text {leaf }} f\right)(p)=4\left\{\sum_{i, j} g_{\alpha \bar{\beta}} g^{\gamma \bar{\delta}} W_{i j ; \gamma}^{\alpha} W_{i j ; \delta}^{\bar{\beta}}-\sum_{i, j} R_{\alpha \beta} W_{i j}^{\alpha} W_{i j}^{\bar{\beta}}\right\}
$$

where $\Delta_{\text {leaf }} f$ is the Laplacian, $R_{\alpha \beta} \bar{\beta}$ are the components of the Ricci tensor of a leaf and $; \gamma$ denotes covariant differentiation in the leaf with respect to the induced connection.

3. Recall that the Ricci tensor of a leaf is called quasi-negative provided it is negative semidefinite on the whole manifold and negative definite at one point of the manifold. With this terminology we state the following result.

THEOREM 3.1. Let $M$ be a compact connected manifold with bundle-like foliation $\mathfrak{T}$. Suppose each leaf of $\mathcal{W}$ is a complex manifold with complex structure induced by restricting to the leaf some globally defined tensor field $J$ on $M$. Suppose that each leaf equipped with this complex structure is Kählerian with respect to the metric and connection induced from $M$ and that local basic vector fields respect the complex structure of the leaves of $\mathcal{V}$.

If the Ricci curvature of each leaf of $\mathcal{T}$ is quasi-negative, then $\mathcal{H}$, the distribution orthogonal to $\mathcal{T}$, is integrable and each leaf of $\mathcal{H}$ is totally geodesic in $M$.

Proof. Observe the hypotheses of the theorem include conditions (A)-(D) of the last section. Let $p \in M$ be the point where $f$ attains its maximum on $M$ and let $L$ be the leaf of $\mathfrak{V}$ passing through $p$. By (10), $\Delta_{\text {leaf }} f \geqslant 0$ on $L$ so $f$ is subharmonic on $L$. It follows for the maximum principle for subharmonic functions that $f$ is constant on $L$ so $\Delta_{\text {leaf }} f=0$ on $L$. Let $q \in L$ be the point where the Ricci tensor of the leaf is negative definite. If $f=c$ and $c \neq 0$, then some $W_{i j}^{\alpha}$ is not identically zero by (9) and so $\left(\Delta_{\text {leaf }} f\right)(q)>0$ again by (10). This contradicts the assertion $\Delta_{\text {leaf }} f=0$ on $L$.

We conclude $f=0$ on $L$ and in particular at $p$. Since $f$ is nonnegative on $M$, our choice of $p$ implies $f=0$ on $M$. This means each $W_{i j}^{\alpha}=0$ by (9) and so $\mathcal{V}\left[X_{i}, X_{j}\right]=0$ by (8). This implies that $\mathscr{V}[X, Y]=0$ for any horizontal $X, Y$ on any open set $U$ and so $\mathcal{H}$ is integrable. It might be mentioned that the argument here was adapted from [8]. 
The fact that the leaves of $\mathcal{H}$ are totally geodesic is immediate from a result of Reinhart which says that a geodesic horizontal at one point is always horizontal. This completes the proof of Theorem 3.1.

We are now in a position to state the main and concluding result of our paper.

Recall a compact Kähler manifold has quasi-negative first Chern class provided this class can be represented by a $(1,1)$ form which is negative semidefinite everywhere and negative definite at a point.

THEOREM 3.2. Let $M$ be a complete connected manifold with bundle-like foliation $\mathfrak{T}$. Suppose that each leaf of $\mathfrak{V}$ is a compact complex manifold whose complex structure is induced by restricting to the leaf some globally defined tensor field J on M. Suppose that each leaf of $\mathfrak{V}$ equipped with this complex structure is Kählerian with respect to the metric and connection induced from $M$ and suppose that the local basic vector fields respect the complex structure of the leaves.

If the first Chern class of each leaf of $\mathcal{V}$ is quasi-negative with respect to one of its representatives, then $\mathcal{H}$, the distribution orthogonal to $\mathcal{T}$ in $T M$, is integrable and each leaf of $\mathcal{H}$ is totally geodesic in $M$.

Proof. To prove this result it suffices to show that $f$ vanishes on each leaf $L$ of $\mathcal{V}$. Suppose the first Chern class of $L$,

$$
c_{1}(L)=\frac{1}{2 \pi \sqrt{-1}} R_{\alpha \bar{\beta}}^{*-} d z^{\alpha} \wedge d \bar{z}^{\bar{\beta}} .
$$

By Yau's solution to the Calabi conjecture [10], there is a metric $g^{*}$ on $L$ so that the Ricci tensor of $g^{*}$ is $R_{\alpha \beta}^{*} d z^{\alpha} \otimes d \bar{z}^{\bar{\beta}}$.

If $i: L \rightarrow M$ is the inclusion mapping on any leaf $L$ of $\mathscr{V}$ then we can modify the metric on the induced bundle $i^{\#}(\mathcal{H}+\mathscr{V})=i^{\#}(\mathcal{H})+i^{\#}(\mathscr{V})$ over $L$ so the metric on $i^{\#}(\mathcal{H})$ is pulled back from that of $\mathcal{H}$ on $M$ and the metric on $i^{\#}(\mathscr{V})$ is $g^{*}$. Since there is no danger of confusion, we identify the sections of $i^{\#}(\mathcal{H}+\mathcal{V})$ with their images in $\mathcal{H}+\mathscr{V}$. Now observe, $\mathscr{V}\left[X_{i}, X_{j}\right]$ is a local automorphism of $J$ where $J$ is the underlying complex structure on $L$.

On the leaf $L$, define the function $h_{L}$ as follows:

$$
h_{L}(p)=\sum_{i, j} 2 g_{\alpha \beta}^{*} W_{i j}^{\alpha} W_{i j}^{\bar{\beta}}
$$

If $\Delta_{\text {leaf }}^{*}$ denotes the Laplacian on $L$ with respect to $g^{*}$, the same reasoning as before yields $\left(\Delta_{\text {leaf }}^{*} h\right) \geqslant 0$ or $h_{L}$ is subharmonic on $L$. Since $L$ is compact, we conclude, as above, $h_{L}=0$ on $L$. Comparing (11) and (9) we are forced to conclude $f=0$ on $L$. Since $L$ was an arbitrary leaf of $\mathfrak{V}$, we see $f=0$ on $M$. This means by (6), that $\mathcal{V}\left[X_{i}, X_{j}\right]=0$ everywhere. Proceeding as in the proof of 3.1 we conclude the proof of Theorem 3.2.

REMARK 3.3. Theorems 3.1 and 3.2 do not imply that $\mathcal{H}$ is holomorphic even if one assumes that $M$ is a Kähler manifold and $\mathcal{V}$ is a holomorphic distribution in TM. See Theorem 2.1 of [3]. 
REMARK 3.4. The condition on the first Chern class of the leaves is necessary as the following example illustrates. Consider

$$
\begin{gathered}
S^{2} \rightarrow \quad C P(2 n+1) \\
\downarrow \pi \\
Q P(n)
\end{gathered}
$$

where $\pi$ is the usual Riemannian submersion [1]. Here $S^{2}$ is the two sphere with constant holomorphic sectional curvature one, $C P(2 n+1)$ is complex projective $2 n+1$ space with constant holomorphic sectional curvature one, while $Q P(n)$ denotes quaternionic projective space. Then this bundle is a special case of the situation where $M=C P(2 n+1)$, each leaf is a copy of $S^{2}$, and $\mathcal{V}$ is the distribution determined by the leaves. It is well known that each leaf of $\mathcal{V}$ is a Kähler submanifold of $C P(2 n+1)$ with respect to the induced complex structure, metric and connection.

To see that basic vector fields respect the complex structure of the leaves of $\mathfrak{V}$, observe that the leaves of $\mathscr{V}$ in this example are totally geodesic and so if $X$ is a basic vector field and $V$ is vertical,

$$
\mathcal{V}[X, J V]=\mathscr{V} \nabla_{X} J X-\mathscr{V} \nabla_{J V} X
$$

Now $J V$ is vertical and hence $\mathfrak{V} \nabla_{J V} X=T_{J V} X=0$, where $T$ is the second fundamental form of the leaves. Since the leaves are totally geodesic, $\mathscr{V} \nabla_{V} X=T_{V} X=0$, and so it follows,

$$
\mathfrak{V}[X, J V]=\mathfrak{V} \nabla_{X} J V=J \mathscr{V} \nabla_{X} V=J \mathscr{V} \nabla_{X} V-J \mathscr{V} \nabla_{V} X=J \mathscr{V}[X, V]
$$

Since $\mathscr{V}[X, J V]=[X, J V]$ and $\mathscr{V}[X, V]=[X, V]$ by Lemma $1.2(\mathrm{a})$, we see that basic vector fields respect the complex structure of the leaves as claimed. However, the distribution $\mathcal{H}$, orthogonal to $\mathscr{V}$ in $C P(2 n+1)$, is not integrable. We note the first Chern class of $S^{2}$ is positive. It follows from this example that the condition on the first Chern class of the leaves in Theorem 3.2 cannot be dropped, if the conclusion of the theorem is to obtain. Observe that in the above theorems unlike this example we do not require that the ambient manifold be Kählerian or even a complex manifold.

ADDED IN PROOF. The above results have some corollaries which will be discussed elsewhere.

\section{REFERENCES}

1. R. H. Escobales, Jr., Riemannian submersions from complex projective space, J. Differential Geom. 13 (1978), 93-107.

2. Bundle-like foliations and the integrability tensor, Trans. Amer. Math. Soc. 270 (1982), 333-339. 71-79.

3. D. L. Johnson, Kähler submersions and holomorphic connections, J. Differential Geom. 15 (1980),

4. S. Kobayashi and K. Nomizu, Foundations of differential geometry. Vol. II, Interscience Tracts in Pure and Applied Mathematics, no. 15, Wiley, New York, 1969.

5. H. B. Lawson, Jr., The quantitative theory of foliations, CBMS Regional Conf. Series in Math., vol. 27, Amer. Math. Soc., Providence, R. I., 1977. 
6. B. O'Neill, The fundamental equations of a submersion, Michigan Math J. 13 (1966), 459-469.

7. B. Reinhart, Foliated manifolds with bundle-like metrics, Ann. of Math. (2) 69 (1959), 119-131.

8. H. Wu, A remark on the Bochner technique in differential geometry, Proc. Amer. Math. Soc. 78 (1980), 403-408.

9. K. Yano and S. Bochner, Curvature and Betti numbers, Ann. of Math. Studies, no. 32, Princeton Univ. Press, Princeton, N. J., 1953.

10. S. T. Yau, On the Ricci curvatures of compact Kähler manifolds and the complex Monge-Ampere equations 1, Comm. Pure Appl. Math 31 (1978), 339-411.

Department of Mathematics, Canisius College, Buffalo, New York 14208 